AcCepted by The Astrophysical Journal Letters

Preprint typeset using $\mathrm{LAT}_{\mathrm{E}} \mathrm{X}$ style emulateapj v. 08/13/06

\title{
FORMATION AND SURVIVABILITY OF GIANT PLANETS ON WIDE ORBITS
}

\author{
EDUARD I. VOROBYOV ${ }^{1,2}$ and SHANTANU BaSU ${ }^{3}$ \\ Accepted by The Astrophysical Journal Letters
}

\begin{abstract}
Motivated by the recent discovery of massive planets on wide orbits, we present a mechanism for the formation of such planets via disk fragmentation in the embedded phase of star formation. In this phase, the forming disk intensively accretes matter from the natal cloud core and undergoes several fragmentation episodes. However, most fragments are either destroyed or driven into the innermost regions (and probably onto the star) due to angular momentum exchange with spiral arms, leading to multiple FU-Ori-like bursts and disk expansion. Fragments that are sufficiently massive and form in the late embedded phase (when the disk conditions are less extreme) may open a gap and evolve into giant planets on typical orbits of several tens to several hundreds of AU. For this mechanism to work, the natal cloud core must have sufficient mass and angular momentum to trigger the burst mode and also form extended disks of the order of several hundreds of AU. When mass loading from the natal cloud core diminishes and the main fragmentation phase ends, such extended disks undergo a transient episode of contraction and density increase, during which they may give birth to a last and survivable set of giant planets on wide and relatively stable orbits.
\end{abstract}

Subject headings: circumstellar matter — planetary systems — protoplanetary disks — hydrodynamics — ISM: clouds — stars: formation

\section{INTRODUCTION}

The likelihood of giant planet formation via direct gravitational instability of circumstellar disks around solar-type stars has been the subject of intense research in the past years. Despite much effort in this field, increasingly sophisticated numerical hydrodynamics simulations and analytical considerations continue to yield conflicting results. On one hand, some studies indicate that giant planets can form in massive disks, particularly in their outer parts where conditions for disk fragmentation are less extreme and the competing core-accretion model is less viable (e.g., Johnson \& Gammie 2003; Stamatellos et al. 2007; Maver et al. 2007; Boss 2008; Dodson-Robinson et al. 2009; Nero \& Bjorkman 2009). On the other hand, many studies show that gravitational fragmentation is unlikely, particularly in the inner few tens of $\mathrm{AU}$ due to insufficient disk cooling and strong stellar/envelope irradiation (e.g., Matzner \& Levin 2005; Rafikov 2005; Bolev et al. 2006, 2007; Rafikov 2007; Stamatellos \& Whitworth 2008; Cai et al. 2008)

In spite of a great deal of sophistication, the aforementioned studies miss one important aspect - circumstellar disks are not isolated in the early embedded phase of star formation (hereafter, EPSF). In this stage, they are subject to intense mass loading from a natal cloud core, which can significantly alter the disk's ability to fragment. A self-consistent handling of this process in numerical simulations is not easy and requires a much larger spatial scale (than just that of the disk). A spatial resolution of less than $1 \mathrm{AU}$ is usually needed for planetary-mass fragments to form and survive. Global

\footnotetext{
${ }^{1}$ Institute for Computational Astrophysics, Saint Mary's University, Halifax, NS, B3H 3C3, Canada; vorobyov@ap.smu.ca.

${ }^{2}$ Research Institute of Physics, Southern Federal University, Stachki 194, Rostov-on-Don, 344090, Russia.

${ }^{3}$ Department of Physics and Astronomy, The University of Western Ontario, London, ON, N6A 3K7, Canada; basu@astro.uwo.ca.
}

numerical hydrodynamics simulations of the gravitational collapse and fragmentation of molecular clouds have demonstrated that forming stars are indeed surrounded by accreting gravitationally unstable disks (e.g., Bate \& Bonnell 2003; Krumholz et al. 2007). Yet, these simulations resolve only massive disks, which, if fragmented, produce brown dwarfs or low-mass stellar companions rather than giant planets. Moreover, such numerical simulations are very computationally intensive and are unable to explore a wide parameter space and long evolution times.

On the other hand, semi-analytic models and simplified numerical simulations of the gravitational collapse of dense cloud cores can explore a wide range of initial conditions and can give us a valuable insight into the required conditions for disk fragmentation. Using the thin-disk approximation, we were able to self-consistently follow the process of cloud core collapse and star/disk formation for at least several Myr after the formation of a central stellar object (Vorobyov \& Basu 2005, 2006, 2009). These studies have shown that circumstellar disks may be gravitationally unstable and susceptible to fragmentation if the rate of gas deposition onto the disk from the cloud core is greater than that from the disk onto the star, disk viscosity is not too high, and the natal cloud cores are characterized by sufficiently large rotation rates. More sophisticated numerical hydrodynamics simulations, though with an approximate treatment of gas infall onto the disk, and semi-analytic studies have confirmed the susceptibility of non-isolated disks to fragmentation, particularly at large radii (e.g., Kratter et al. 2008; Rice at al. 2009; Boley 2009; Boley et al. 2009; Clarke 2009; Rafikov 2009).

The feasibility of disk fragmentation and giant planet formation is only one part of the problem. The other part is the likelihood of survival of giant planets formed via disk fragmentation. Rapid radial migration due to gravitational interaction of a giant planet with a natal 
gas disk Goldreich \& Tremaine 1980) has traditionally been one of the stumbling blocks for the theory of giant planet formation and many mechanisms have been proposed to stop this migration in the late evolution phase (see e.g., Thommes \& Murray 2006; Crida \& Morbidelli 2007; Ida \& Lin 2008; Matsumura et al. 2009). In the early EPSF, this problem may be even more severe due to the fact that disks are more massive and profoundly non-axisymmetric. Indeed, our previous numerical studies have shown that fragments forming in the EPSF are quickly driven into the inner regions and probably onto the protostar due to exchange of angular momentum with spiral arms (Vorobyov \& Basu 2005, 2006). We have speculated that only those fragments that form in the late EPSF, when gravitational instability starts to gradually decline with time, may have a chance to survive.

In this paper, we present confirmation that the fragments formed in the EPSF can survive through this extreme phase and form giant protoplanets (hereafter, GPPs) on large, relatively stable orbits. This finding is made possible by the employment of expanded computational resources, by improvements in the numerical model, and by the use of a wider parameter space in comparison to previous works.

\section{MODEL EQUATIONS AND INITIAL CONDITIONS}

Our numerical model is explained in detail in Vorobyov \& Basu (2006) and is briefly summarized below. We make use of the thin-disk approximation to compute the gravitational collapse of rotating, gravitationally unstable cloud cores. We start our numerical integration in the pre-stellar phase, which is characterized by a collapsing starless cloud core with a typical radius of $10^{4} \mathrm{AU}$, continue into the EPSF, which sees the formation of a star/disk/envelope system, and terminate our simulations in the late $\mathrm{T}$ Tauri phase. The mass accretion rate onto the disk, $\dot{M}_{\text {env }}$, is not a free parameter but is self-consistently determined by the dynamics of the gas in the envelope. We introduce a "sink cell" at $r_{\mathrm{sc}}=8 \mathrm{AU}$ and impose a free inflow inner boundary condition. Ninety per cent of the gas that crosses the inner boundary is assumed to land onto the central star plus the inner axisymmetric disk at $r<8 \mathrm{AU}$. The other $10 \%$ of the accreted gas is assumed to be carried away with protostellar jets.

The basic equations of mass and momentum transport in the thin-disk approximation are

$$
\begin{aligned}
\frac{\partial \Sigma}{\partial t} & =-\nabla_{p} \cdot\left(\Sigma \boldsymbol{v}_{p}\right) \\
\Sigma \frac{d \boldsymbol{v}_{p}}{d t} & =-\nabla_{p} \mathcal{P}+\Sigma \boldsymbol{g}_{p}+(\nabla \cdot \boldsymbol{\Pi})_{p}
\end{aligned}
$$

where $\Sigma$ is the mass surface density, $\mathcal{P}=\int_{-Z}^{Z} P d z$ is the vertically integrated gas pressure, $Z$ is the vertical scale height, $\boldsymbol{v}_{p}=v_{r} \hat{\boldsymbol{r}}+v_{\phi} \hat{\boldsymbol{\phi}}$ is the velocity in the disk plane, $\boldsymbol{g}_{p}=g_{r} \hat{\boldsymbol{r}}+g_{\phi} \hat{\boldsymbol{\phi}}$ is the gravitational acceleration in the disk plane, and $\nabla_{p}=\hat{\boldsymbol{r}} \partial / \partial r+\hat{\boldsymbol{\phi}} r^{-1} \partial / \partial \phi$ is the gradient along the planar coordinates of the disk. The gravitational acceleration $\boldsymbol{g}_{p}$ includes the gravity of a central point object (when formed), the gravity of the inner inactive disk $(r<8 \mathrm{AU})$, and the self-gravity of a circumstellar disk and envelope. For the kinematic viscosity $\nu$ that enters the viscous stress tensor $\boldsymbol{\Pi}$, we use the usual $\alpha$-prescription, with a spatially and temporally uniform $\alpha=0.005$. The latter choice is based on our recent work (Vorobyov \& Basu 2009), i.e., $\alpha$ is small enough to not eliminate the burst mode and large enough to drive significant accretion in the late stages of disk evolution.

Equations (11) and (2) are closed with a barotropic equation that makes a smooth transition from isothermal to adiabatic evolution at $\Sigma=\Sigma_{\text {cr }}$ (Vorobyov \& Basu 2006). For the ratio of specific heats we use $\gamma=1.4$. The $\gamma=5 / 3$ case was explored in Vorobyov \& Basu (2006). The value of $\Sigma_{\mathrm{cr}}$ is calculated during the numerical simulations as $\Sigma_{\mathrm{cr}}=m_{\mathrm{H}} \mu n_{\mathrm{cr}} 2 Z$, where the critical volume number density $n_{\text {cr }}$ is set to $10^{11} \mathrm{~cm}^{-3}$ (Larson 2003) and the mean molecular weight $\mu=2.33$. The scale height $Z$ is calculated using the assumption of vertical hydrostatic equilibrium. We note that $Z$ is an increasing function of radius, which makes $\Sigma_{\text {cr }}$ increase with radius as well. In practice, this means that the inner disk regions are significantly warmer than the outer regions, since the optically thick regime in the inner regions is achieved at lower $\Sigma$. This in turn impedes the development of gravitational instability and fragmentation in the inner disk, in agreement with more sophisticated numerical simulations and theoretical predictions that directly solve for the energy balance equation (see e.g., Stamatellos \& Whitworth 2008; Boley 2009; Boley et al. 2009 ). The use of a spatially varying $\Sigma_{\text {cr }}$ is an important improvement of the numerical model as compared to our previous works. Equations (11) and (2) are solved in polar coordinates $(r, \phi)$ on a numerical grid with $256 \times 256$ or $512 \times 512$ (depending on the model) grid zones using the method of finite-differences. The radial points are logarithmically spaced.

Initially, cloud cores have surface densities $\Sigma$ and angular velocities $\Omega$ typical for a collapsing, axisymmetric, magnetically supercritical core (Basu 1997), with $\Sigma, \Omega \propto$ $r^{-1}$ at large radii. Cloud cores are initially isothermal and they are characterized by a specific ratio of the rotational to gravitational energy $\beta=E_{\text {rot }} /\left|E_{\text {grav }}\right|$. We have considered the evolution of 82 cloud cores with masses $M_{\mathrm{cl}}=0.2-3.0 M_{\odot}$, energy ratios $\beta=0.2-2.2 \times 10^{-2}$, and initial gas temperatures $T=10-18 \mathrm{~K}$. The initial column density is flattened near the center and achieves an asymptotic large-radius profile $\Sigma(r)=k c_{\mathrm{s}}^{2} /(G r)$, where $k=\sqrt{A} / \pi$. In our previous papers (e.g. Vorobyov 2009), we took $A=2$, but here let it vary in the range $A=2-8$, so that models can be more gravitationally unstable.

\section{FORMATION OF GIANT PLANETS}

\subsection{Conditions for fragmentation}

The formation of giant planets via disk fragmentation can only take place if the the ratio of the local cooling time $t_{\mathrm{c}}$ to the local dynamical time $\Omega^{-1}$ is smaller than a few (e.g., Gammie 2001; Rice at al. 2003; Meiía et al. 2005). Our preliminary results with disk cooling, viscous and shock heating, and stellar irradiation included in the code indicate that this condition is satisfied in the EPSF, at least in the disk's outer regions (Vorobyov \& Basu 2010).

Another requirement for disk fragmentation is the Toomre criterion $Q=c_{\mathrm{S}} \Omega /(\pi G \Sigma)<1$, which states that 
the gas surface density $\Sigma$ should be sufficiently high for a disk to fragment. Too high a $\Sigma$, however, may prevent fragmentation due to increased opacity and cooling time (Nero \& Bjorkman 2009). In other words, there exists minimum and maximum values of $\Sigma$ between which the instability and fragmentation are expected to occur. In numerical simulations that form disks self-consistently (such as our own), $\Sigma$ naturally increases from low toward higher values during the disk formation phase and the disk may pass through the unstable regime.

The critical density for fragmentation can in principle be achieved if the rate of mass deposition onto the disk $\dot{M}_{\text {env }}$ is greater than the mass flux in the disk $\dot{M}_{\mathrm{d}}$ so that $\Sigma$ quickly increases with time (Vorobvov \& Basu 2007; Vorobvov 2009; Kratter et al. 2009; Bolev 2009) and this phase of intense infall (i.e., the EPSF) lasts for many dynamical times (so that the GPPs have enough time to form). Simple analytical estimates for a viscous disk indicate that $\dot{M}_{\mathrm{d}} / \dot{M}_{\text {env }} \approx 3 \alpha / Q$ for $T=30 \mathrm{~K}$ Boley 2009), which implies that the outer disk regions are expected to fragment for values of $\alpha \sim 10^{-2}$. Numerical simulations by Vorobyov (2009) also show that $\dot{M}_{\text {env }}$ is on average several times greater than $\dot{M}_{\mathrm{d}}$ in the EPSF.

Observations provide conflicting estimates as to the duration of the embedded phase $\tau_{\mathrm{em}}$, ranging from a few $\times 10^{4}$ yr to a few $\times 10^{5}$ yr André \& Montmerle 1994; Evans et al. 2009). From simple analytical grounds it follows that $\tau_{\mathrm{em}}$ should be linearly proportional to the initial cloud core mass $M_{\mathrm{cl}}$ and inverse proportional to $T^{3 / 2}$ and $A$. In addition, such effects as rotation and magnetic fields may steepen the relationship, particularly for cloud cores of solar mass and greater. The numerically obtained values for $\beta=1.3 \times 10^{-2}, T=10 \mathrm{~K}$, and $A=2$ range from $0.03 \mathrm{Myr}$ for $M_{\mathrm{cl}}=0.2 M_{\odot}$ to $0.5 \mathrm{Myr}$ for $M_{\mathrm{cl}}=2.1 M_{\odot}$ (Vorobyov 2010). These values may decrease by as much as $3-4$ for $T=20 \mathrm{~K}$ or $A=8$. Nevertheless, cloud cores in the aforementioned mass range are expected to have $\tau_{\mathrm{em}}$ that are considerably longer than typical orbital times of 360-3200 yr for radial distances of 50-100 AU and stellar masses of 0.1-1 $M_{\odot}$.

Another important initial parameter that determines the disk propensity to fragment is the amount of rotation in the natal cloud core. Indeed, cloud cores with greater rotation rates would make larger disks since the centrifugal radius is proportional to the square of the specific angular momentum, which would increase disk tendency to fragment (recall that fragmentation tends to occur at large radii). This has been confirmed by numerical and semi-analytic simulations of disk formation (Vorobvov \& Basu 2006; Kratter et al. 2008; Vorobvov 2009; Rice at al. 2009).

The above analysis indicates that disks formed from sufficiently massive cloud cores with sufficiently high angular momenta are expected to fragment in the embedded phase, particularly in the outer regions. This conclusion has been corroborated by numerical simulations that form disks self-consistently (Vorobvov \& Basu 2005, 2006, 2009; Vorobyov 2009; Kratter et al. 2009) or impose some prescribed rate of mass infall onto the disk (Boley 2009; Boley et al. 2009; Rice at al. 2009). It is therefore not so much the feasibility of fragmentation as the likelihood of survival of the forming fragments that we focus in the present study.

\subsection{Numerical results}

In agreement with Section 3.1 we have found that the disk propensity to fragment increases along the sequence of increasing $M_{\mathrm{cl}}$ and $\beta$. This tendency is reflected in both the greater numbers and higher masses of the fragments, which form via fragmentation of dense spiral arms. The final masses of fragments that form in our simulations lie in a wide range starting from a few Jupiter masses and ending with low- and intermediatemass brown dwarfs. For disk fragmentation to take place, the minimum cloud core mass should be $M_{\mathrm{cl}, \min } \approx$ $0.8 M_{\odot}$ for $\beta=0.2 \times 10^{-3}$ and $A=2$. For $\beta=2 \times 10^{-2}$ and $A=2$, the corresponding $M_{\mathrm{cl}, \min }$ is approximately $0.2 M_{\odot}$. These minimum masses decrease by about $30 \%$ for higher perturbation amplitudes $A=8$ due to an associated increase in $\dot{M}_{\text {env }}$, which promotes disk fragmentation. On the other hand, our preliminary results suggest that $M_{\mathrm{cl} \text {,min }}$ may increase somewhat if a more accurate treatment of the thermal physics is considered.

Not all fragments evolve ultimately to GPPs, most have either migrated through the inner boundary or dispersed in the outer regions (possibly due to insufficient resolution). Only 6 out of the 82 models have formed GPPs with final masses in the $M_{\mathrm{pl}}=5-10 M_{\mathrm{J}}$ range on relatively stable orbits at 25-200 AU. In two models we saw two GPPs forming simultaneously, but in both cases the outer one had dispersed just after 1.0 Myr of evolution, either due to insufficient numerical resolution or tidal disruption by the inner GPP because of proximity to the 4:1 resonance. These numbers should not be treated as representative, since we expect the number of survived GPPs to increase in simulations with a higher numerical resolution.

Among 47 models with $\beta<10^{-2}$ only two models with high density perturbation amplitude $A=8$ (as opposed to standard $A=2$ ) have revealed planet formation. The most likely reason why low- $\beta$ and low- $A$ models fail to form GPPs is that their disks are too small and the fragments are forming too close to the star, which lowers their chances to survive. In addition, these models have lower disk-to-star mass ratios than their high- $\beta$ and high$A$ counterparts, which results in the formation of lowermass fragments. Such fragments need more time to open the gap and slow down the fast inward migration in the embedded phase.

Figure 1 presents the gas surface density maps (in $\left.\mathrm{g} \mathrm{cm}^{-2}\right)$ in model $1\left(M_{\mathrm{cl}}=0.9 M_{\odot}, \beta=1.3 \times 10^{-2}\right.$, $A=2$ ) at six evolution times after the formation of the disk. The rotation is counterclockwise (note that we zoom in at the bottom row). Several fragments condense in the outer parts of the spiral arms as early as $0.09 \mathrm{Myr}$ after the disk formation, but none of them have survived by the end of the embedded phase at $\approx 0.16 \mathrm{Myr}$ when about $75-80 \%$ of the envelope has been accreted by the disk. They are all driven into the sink cell via a very efficient exchange of angular momentum with the spiral arms, possibly leading to multiple FU Orionis bursts (Vorobyov \& Basu 2005, 2006) or forming giant planets on very close orbits. The byproduct of these bursts is disk expansion due to the conservation of angular momentum. When the mass loading from the envelope diminishes and the burst phase ends, this expansion is followed by transient disk contraction, during which gas surface density 


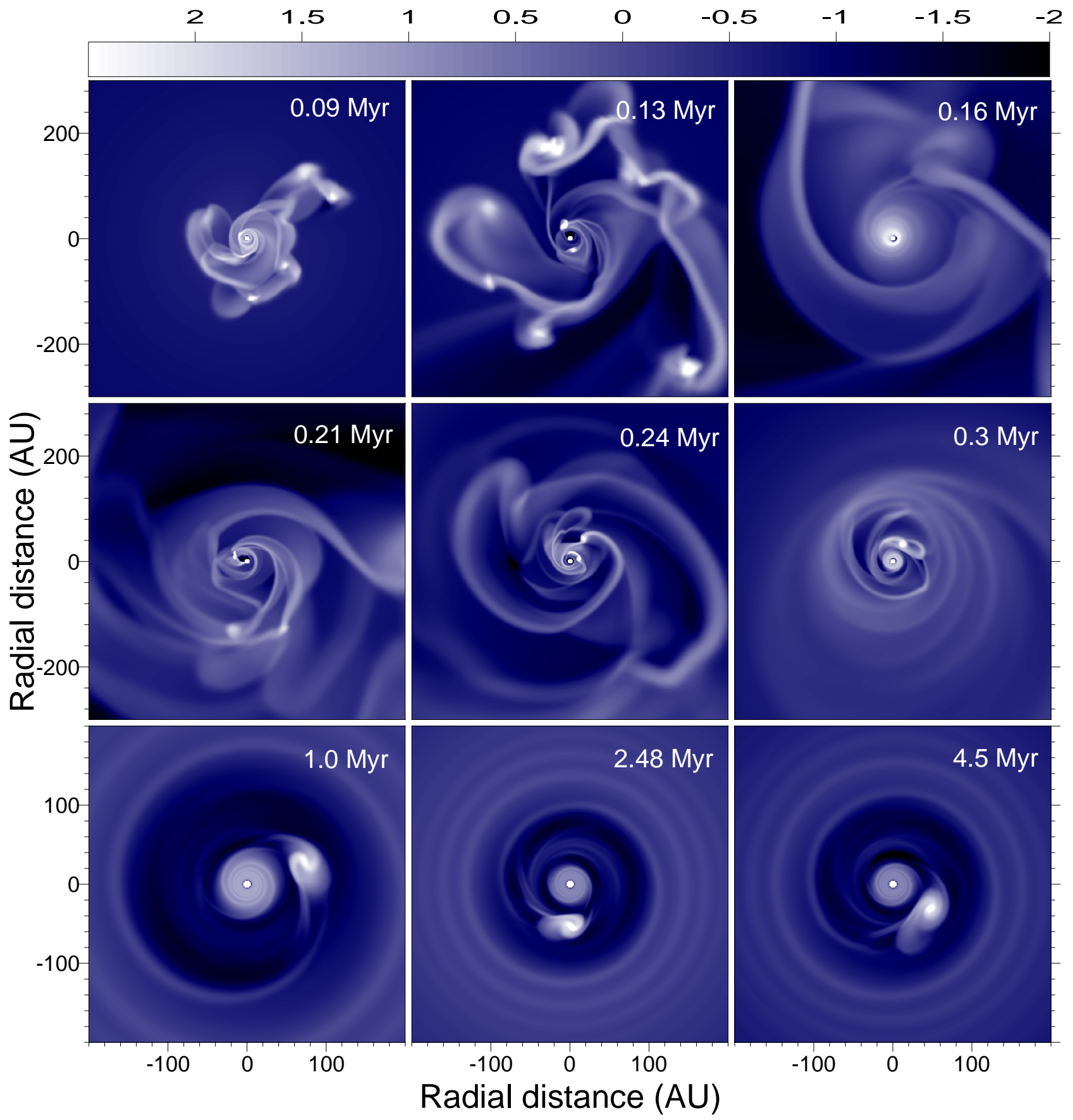

FIG. 1.- Gas surface density maps $\left(\mathrm{g} \mathrm{cm}^{-2}, \log\right.$ units) at six times after the formation of the central star (bright circle in the coordinate center) in model $1\left(M_{\mathrm{cl}}=0.9 M_{\odot}, \beta=1.3 \times 10^{-2}, A=2\right)$. Note that we zoom in as the time increases. The top two rows contain images of size $600 \mathrm{AU}$ on each side, while the bottom row contains images of size $400 \mathrm{AU}$ on each side.

increases and several more fragments form in the disk's outermost regions $(t=0.21 \mathrm{Myr})$. These fragments quickly migrate in the inner $100 \mathrm{AU}$ and, by $t=0.3 \mathrm{Myr}$, only one fragment survives, which later opens a gap and evolves into a well defined GPP possessing its own counterrotating minidisk. Such counterrotating minidisks are seen around many fragments. We believe that this effect is caused by the gravitational capture of some of the neighboring material, which receives a counterrotating twist around the forming fragment due to differential rotation of the natal spiral arm.

Figure 2 shows the GPP's radial position $r_{\mathrm{pl}}$ (top), mass (middle), and Hill's radius (bottom), as a function of time in model 1. Since we do not use sink particles for GPPs, these values should be treated as approximate. Upon its formation at $r_{\mathrm{p}} \gtrsim 100 \mathrm{AU}$, the GPP migrates quickly in the inner $40 \mathrm{AU}$ and back (this time more slowly) to $r \approx 80$ AU. The outward migration is then followed by a gradual inward migration until the GPP finally settles at $r_{\mathrm{p}} \approx 52 \mathrm{AU}$.

The mass of the GPP is estimated by integrating the azimuthally-averaged gas surface density profile around a local maximum at the planet location. The middle panel of Fig. 2 shows the upper limits on the total mass of the inner GPP plus its mini-disk (dashed line) and the mass $M_{\mathrm{pl}}$ of the inner GPP (solid line). The latter value is 


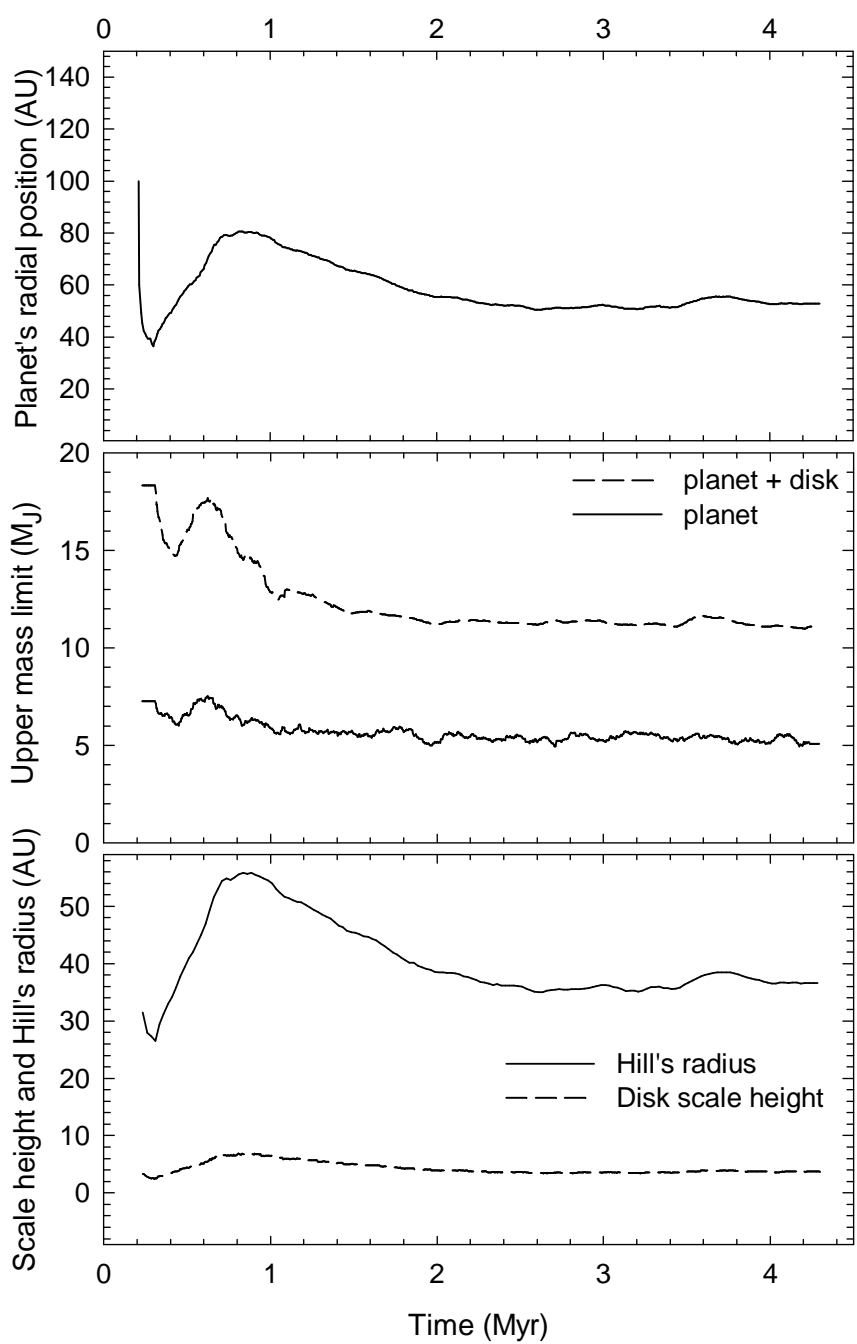

Fig. 2.- Planet's radial position (top), mass (middle), and Hill's radius and vertical scale height (bottom), for model 1 .

uncertain to within a factor of unity. The total (planet plus disk) mass is always in the giant planet regime, while $M_{\mathrm{pl}}$ is around $5 \pm 1 M_{\mathrm{J}}$. The bottom panel shows the Hill radius $r_{\mathrm{H}}=r_{\mathrm{pl}}\left(M_{\mathrm{pl}} / 3\left(M_{*}+M_{\mathrm{pl}}\right)\right)^{1 / 3}$ of the GPP (solid line) and the azimuthally-averaged vertical scale height at the planet position $\bar{Z}$ (dashed line). It is seen that a condition for gap opening, $r_{\mathrm{H}}>\bar{Z}$, is satisfied.

How stable are the orbits of GPPs in our modeling? Figure 3 presents the radial position of GPPs vs. time in model 2 (solid line, $M_{\mathrm{cl}}=0.4 M_{\odot}, \beta=0.01, A=2$ ) and model 3 (dashed line, $M_{\mathrm{cl}}=2.0 M_{\odot}, \beta=3 \times 10^{-3}$, $A=8$ ). Initially, model 2 demonstrates a similar migration pattern to that of model 1 . However, the GPP in model 2 does not seem to settle at a stable orbit but slowly migrates inward. Whether or not this migration would stop at later times is unclear. On the contrary, the GPP in model 3 appears to stabilize at a rather large radial distance of $190 \mathrm{AU}$. These examples indicate that GPPs may have various migration histories, which depend probably on particular physical conditions in the disk and the natal cloud core. The net planet masses are $5 \pm 1 M_{\mathrm{J}}$ in model 2 and $10 \pm 1 M_{\mathrm{J}}$ in model 3 .

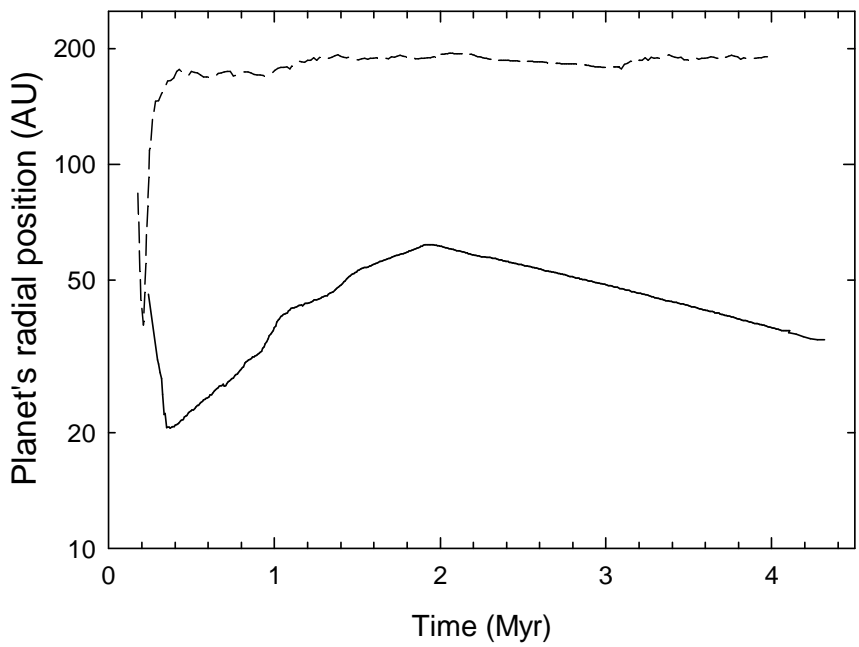

FIG. 3.- Planet's radial position in model 2 (solid line, $M_{\mathrm{cl}}=$ $0.4 M_{\odot}, \beta=0.01, A=2$ ) and model 3 (dashed line, $M_{\mathrm{cl}}=2.0 M_{\odot}$, $\beta=3 \times 10^{-3}, A=8$ ).

We have studied the long-term evolution of disks that are formed by the self-consistent collapse of prestellar cores. Our model yields gas giant formation starting from initial conditions of the early stages of star formation. The initial cores are more gravitationally unstable and have greater angular momenta than similar models studied in the past, and a large number of models have been run with relatively high resolution. An early burst mode of evolution is characterized by the formation of clumps which are then driven into the inner disk. However, in a small subset of models, massive fragments are formed on wide orbits and settle into stable orbits of radius $\gtrsim 50 \mathrm{AU}$. An interesting feature is that minidisks around these fragments can be counterrotating with respect to the disk. Sometimes, the final orbit can be much larger or alternatively there can be a slowly continuing inward migration. We believe that our results can explain the purported observations of giant planets on wide orbits. By extrapolation, they may also represent the first stages of the eventual formation of a low mass brown dwarf companion.

We are thankful to the referee, Dr. Richard Durisen, for an insightful report. EIV gratefully acknowledges present support from an ACEnet Fellowship. SB was supported by a grant from NSERC. Numerical simulations were done on the Atlantic Computational Excellence Network (ACEnet), on the Shared Hierarchical Academic Research Computing Network (SHARCNET), and at the Center of Collective Supercomputer Resources, Taganrog Technological Institute, South Federal University.

\section{REFERENCES}

André, P., \& Montmerle, T. 1994, ApJ, 420, 837

Basu, S. 1997, ApJ, 485, 240

Bate, M. R., Bonnell, I. A. \& Bromm, V. 2003, MNRAS, 339, 577
Boley, A. C., Mejía, A. C., Durisen, R. H., Cai, K., Pickett, M. K., \& D'Alessio, P. 2006, ApJ, 651, 517 
Boley, A. C., Durisen, R. H., Nordlung, A., \& Lord, J. 2007, ApJ, 665,1254

Boley, A. C. 2009, ApJ, 695, L53

Boley, A. C., Hayfield, T., Mayer, L., \& Durisen, R. H. 2009, astroph:0909.4543

Boss, A. P. 2008, ApJ, 677, 607

Cai, K., Durisen, R. H., Boley, A. C., Pickett, M. K., \& Mejía, A. C. $2008, \mathrm{ApJ}, 673,1138$

Clarke, C. J. 2009, MNRAS, 396, 1066

Crida, A., \& Morbidelli, A. 2007, MNRAS, 377, 1324

Dodson-Robinson, S. E., Veras, D., Ford, E. B., \& Beichman, C. A. 2009, ApJ, 707, 79

Evans II, N. J., Dunham, M. M., Jorgensen, J. K., Enoch, M. L., Merín, B., van Dishoeck, E. F., et al. 2009, ApJS, 181, 321

Gammie, C. F. 2001, ApJ, 553, 174

Goldreich, P., \& Tremaine, S. 1980, ApJ, 241, 425

Ida, S., \& Lin, D. N. C. 2008, ApJ, 673, 487

Johnson, B. M. \& Gammie, C. F. 2003, ApJ, 597, 131

Kratter, K. M., Matzner, C. D., \& Krumholz, M. R. 2008, ApJ, 681,375

Kratter, K. M., Matzner, C. D., Krumholz, M. R., \& Klein, R. I. 2009, astro-ph:0907.3476

Krumholz, M. R., Klein, R. I., \& McKee, C. F. 2007, ApJ, 656, 959 Larson, R. B., 2003, Rep. Prog. Phys., 66, 1651

Matzner, C. D., \& Levin, Yu. 2005, ApJ, 628, 817

Mayer, L., Lufkin, G., Quinn, T., \& Wadsley J. 2007, ApJ, 661, L77
Mejía, A. C., Durisen, R. H., Pickett, M. K., \& Cai, K. 2005, ApJ, 619,1098

Nakamoto, T., \& Nakagawa, Y. 1994, ApJ, 421, 640

Nero, D., \& Bjorkman, J. E. 2009, ApJ, 702, L163

Rafikov, R. R., 2005, ApJ, 621, 69

Rafikov, R. R. 2007, ApJ, 662, 642

Rafikov, R. R. 2009, ApJ, 704, 281

Rice, W. K. M., Armitage, P. J., Bate, M. R., \& Bonnell, I. A. 2003, MNRAS, 339, 1025

Rice, W. K. M., Mayo, J. H., \& Armitage, P. J. 2009, astroph:0911.1202

Shu, F. H. 1977, ApJ, 214, 488

Matsumura, S., Pudritz, R. E., Thommes, E. W. 2009, ApJ, 691, 1764

Stamatellos, D., Hubber, D. A., \& Whitworth, A. P. 2007, MNRAS, 382,30

Stamatellos, D., \& Whitworth, A. P. 2008, A\&A, 480, 879

Thommes, E., \& Murray, N. 2006, ApJ, 644, 1214

Vorobyov, E. I., \& Basu, S. 2005, ApJ, 633, L137

Vorobyov, E. I., \& Basu, S. 2006, ApJ, 650, 956

Vorobyov, E. I., \& Basu, S. 2007, MNRAS, 381, 1009

Vorobyov, E. I., \& Basu, S. 2009, MNRAS, 393, 822

Vorobyov, E. I., \& Basu, S. 2010, in preparation

Vorobyov, E. I. 2009, ApJ, 704, 715

Vorobyov, E. I. 2010, ApJ, 713, 1 